\title{
Estadística de egr esos hospitalarios del sector público del Sistema Nacional de Salud, 2002
}

L

a Dirección General de Información en Salud, entre otras funciones, se constituye como la ventanilla única responsable de integrar y difundir la información sobre la producción de servicios de salud de la Secretaría de Salud, así como responsable de integrar, en este mismo apartado, toda la información del sector tanto público como privado; además, con la intención de dar un giro, en su acción cotidiana, prestando mayor atención a la mejoría en la calidad y veracidad de los datos, lo que permitirá mayor solidez en el procesamiento y así pasar de un área generadora de bases de datos, a una generadora de información que permita la toma de decisiones y apoye la gestión en los distintos niveles de la organización del sistema de salud.

No obstante, este nuevo sentido de la Dirección General no pretende, en ningún momento, dejar de lado su función primordial de recopilación de la información; por ello, y con la finalidad de dar continuidad a la información que se ofrece sobre egresos, se presenta la correspondiente al año 2002.

En esta ocasión se elaboraron los cuadros de información estadística con un cambio en la clasificación y presentación de las principales cau- sas, tanto de morbilidad como de mortalidad, lo cual permite incluir a todos los diagnósticos y agrupar los egresos en tres grandes grupos: enfermedades transmisibles, maternas, perinatales y nutricionales; enfermedades no transmisibles, y lesiones, considerando dos apartados: otras causas de contacto con los servicios de salud y causas mal definidas. Esta propuesta se está afinando y enriqueciendo con las aportaciones de los usuarios de dicha información; por otro lado, se modifica la agrupación por edad y se desglosa para menores de un año de edad (menores de siete días, de ocho a 28 días y de un mes a un año).

Por el tipo de modificaciones planteadas, para el presente se incluye la información de la Secretaría de Salud, el Instituto Mexicano del Seguro Social, el Instituto de Seguridad y Servicios Sociales de los Trabajadores del Estado, la Secretaría de la Defensa Nacional y el IMSS-Oportunidades, y se excluyen la de Petróleos Mexicanos y Secretaría de

\begin{tabular}{|c|c|c|c|c|c|c|}
\hline \multirow[b]{3}{*}{ Egresos total } & \multicolumn{3}{|c|}{ EGRESOS HOSPITALARIOS } & & & \\
\hline & Hombres & M ujeres & Total & \multicolumn{3}{|c|}{ Porcentajes } \\
\hline & 1317487 & 2900228 & 4217715 & 31.2 & 68.8 & 100.0 \\
\hline No derechohabiente & 483372 & 1362931 & 1846303 & 36.7 & 46.9 & 43.8 \\
\hline SSA & 440064 & 1195237 & 1635301 & & & \\
\hline IMSS-0 portunidades & 43308 & 167694 & 211002 & & & \\
\hline Derechohabiente & 834115 & 1537297 & 2371412 & 63.3 & 53.0 & 56.2 \\
\hline IMSS & 681702 & 1266922 & 1948624 & & & \\
\hline ISSSTE & 116101 & 220907 & 337008 & & & \\
\hline Sedena & 36312 & 49468 & 85780 & & & \\
\hline \multicolumn{7}{|c|}{$\begin{array}{l}\text { SSA: Secretaría de Salud } \\
\text { IMSS: Instituto Mexicano del Seguro Social } \\
\text { ISSSTE: Instituto de Seguridad y Servicios Sociales de los Trabajadores del Estado } \\
\text { Sedena: Secretaría de la D efensa N acional }\end{array}$} \\
\hline
\end{tabular}

Sección preparada por la Dirección General de Información y Evaluación del Desempeño. Secretaría de Salud. México. 
Marina, cuyas bases están en revisión conforme con las modificaciones descritas. Queda aún como saldo pendiente incluir la información del sector privado.

Actualmente las cifras de egresos del sector público superan los 4.2 millones de egresos, casi $69 \%$ de mujeres y poco más de $56 \%$ de derechohabientes

Se presentan 16 cuadros estadísticos, ocho de morbilidad y ocho de mortalidad, considerando el mismo tipo de información para ambas bases. Los primeros cuatro* informan por población derechohabiente y no derechohabiente y diferencian entre hombres y mujeres, con una adaptación mexicana de la lista manejada por la Organización Mundial de la Salud; es interesante observar cómo las causas por enfermedades no transmisibles, para el sexo masculino representan cerca de $60 \%$, las mujeres el porcentaje desciende a $34 \%, \$$ por la variable "causas maternas"; este dato representa, en el caso de derechohabientes, $81 \%$ de los egresos por enfermedades transmisibles, maternas, perinatales y nu- tricionales y casi 37\% de los egresos totales, mientras que en el caso de no derechohabientes los porcentajes son de 89 y $62 \%$, respectivamente, lo que por su variabilidad también constituye un dato digno de ser analizado.

Los siguientes dos cuadros informan por institución y por sexo; ${ }^{1 \#}$ finalmente se clasifican los egresos por tipo de edad y sexo (y se destaca la desagregación para menores de un año). ${ }^{*}$

* Cuadros I, II, III y IV para morbilidad, y XI, X, XI y XII para mortalidad

‡ $64 \%$ para población derechohabiente y $53 \%$ para no derechohabiente

$\S 44 \%$ para población derechohabiente y $24 \%$ para no derechohabiente

* Cuadros V y VI para morbilidad, y XIII y XIV para mortalidad

\& Cuadros VII y VIII para morbilidad, y XV y XVI para mortalidad

* Cuadros V y VI para morbilidad, y XIII y XIV para mortalidad 


\section{Cuadro I \\ Grandes grupos de causas de morbilidad hospitalaria, población derechohabiente.* Hombres, MÉxico, 2002}

Causas

Total
Egresos

834115

145771

Enfermedades transmisibles, maternas, perinatales y nutricionales

Enfermedades infecciosas y parasitarias

Infecciones respiratorias

Ciertas afecciones originadas en el periodo perinatal

Deficiencias de la nutrición

39042

54013

4894
Tasa por $100000^{\ddagger}$

3123

100.0

\begin{tabular}{lrrr}
\hline Enfermedades no transmisibles & 531749 & 1991 & 63.8 \\
\hline Tumores malignos & 38972 & 146 & 4.7 \\
\hline O tros tumores & 11491 & 43 & 1.4 \\
\hline Diabetes mellitus & 37864 & 142 & 4.5 \\
\hline Enfermedades endócrinas, metabólicas, hematológicas e inmunológicas & 9596 & 36 & 1.2 \\
\hline Trastornos mentales y enfermedades del sistema nervioso central & 20189 & 76 & 2.4 \\
\hline Enfermedades de los órganos de los sentidos & 13817 & 52 & 1.7 \\
\hline Enfermedades cardiovasculares & 77448 & 290 & 9.3 \\
\hline Enfermedades respiratorias & 43776 & 164 & 5.2 \\
\hline Enfermedades digestivas & 119683 & 448 & 14.3 \\
\hline Enfermedades del sistema genito-urinario & 87848 & 329 & 10.5 \\
\hline Enfermedades de la piel & 33644 & 126 & 4.0 \\
\hline Enfermedades del sistema músculo-esquelético & 17410 & 65 & 2.1 \\
\hline Anomalías congénitas & 18247 & 68 & 2.2 \\
\hline Enfermedades de la boca & 1764 & 7
\end{tabular}

\begin{tabular}{lrrr} 
Lesiones & 101606 & 380 & 12.2 \\
\hline Fracturas & 49587 & 186 & 5.9 \\
\hline Amputación traumática & 2090 & 8 & 0.3 \\
\hline Luxaciones, esguinces y desgarros & 4962 & 19 & 0.6 \\
\hline Heridas & 6783 & 25 & 0.8 \\
\hline Q uemaduras & 3961 & 0.5 & 0.2 \\
\hline Envenenamientos & 1796 & 121 & 3.9 \\
\hline O tros traumatismos & 32427 & 127 & 4.1 \\
O tras causas de contacto con los servicios de salud & 33882 & 79 & 2.5
\end{tabular}

Causas mal definidas

21107

\begin{tabular}{rr}
546 & 17.5 \\
\hline 146 & 4.7 \\
\hline 179 & 5.7 \\
\hline 18 & 6.5 \\
\hline
\end{tabular}

* Incluye: Instituto Mexicano del Seguro Social, Instituto de Seguridad y Servicios Sociales de los Trabajadores del Estado y Secretaría de la Defensa Nacional

‡ Estimación realizada a partir de la muestra censal del Censo 2000 y las proyecciones de la población de México 2000-2030. Consejo Nacional de Población, 2002

Fuente: Secretaría de Salud. Dirección General de Información y Evaluación del Desempeño. Base de datos de Egresos Hospitalarios. México, 2002 


\section{Cuadro II

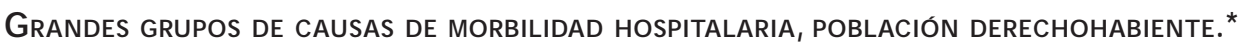 Mujeres, México, 2002}

Causas

Total
Egresos

1537297

716353

Enfermedades transmisibles, maternas, perinatales y nutricionales

Enfermedades infecciosas y parasitarias

Infecciones respiratorias

Causas maternas

Ciertas afecciones originadas en el periodo perinatal

Deficiencias de la nutrición

Enfermedades no transmisibles

\begin{tabular}{l}
\hline Tumores malignos \\
\hline O tros tumores \\
\hline Diabetes mellitus \\
\hline
\end{tabular}

Enfermedades endócrinas, metabólicas, hematológicas e inmunológicas

Trastornos mentales y enfermedades del sistema nervioso central

Enfermedades de los órganos de los sentidos

Enfermedades cardiovasculares

Enfermedades respiratorias

Enfermedades digestivas

Enfermedades del sistema genito-urinario

Enfermedades de la piel

Enfermedades del sistema músculo-esquelético

Anomalías congénitas

Enfermedades de la boca

156901

128780

35633

19696

15882

2002

\begin{tabular}{rrr}
716353 & 2627 & 46.6 \\
\hline 44210 & 162 & 2.9 \\
\hline 38733 & 142 & 2.5 \\
\hline 583328 & 2139 & 37.9 \\
\hline 43489 & 159 & 2.8 \\
\hline 6593 & 24 & 0.4
\end{tabular}

$\begin{array}{lrr}681100 & 2497 & 44.3\end{array}$

$\begin{array}{lll}47348 & 174 & 3.1\end{array}$

$58889 \quad 216 \quad 3.8$

$44799 \quad 164 \quad 2.9$

$14125 \quad 52 \quad 0.9$

$\begin{array}{lll}22230 & 82 & 1.4\end{array}$

$15390 \quad 56 \quad 1.0$

$79295 \quad 291 \quad 5.2$

$\begin{array}{lrr}40130 & 147 & 2.6\end{array}$

575

575
472
131

131

131

58

64980

33674

\begin{tabular}{l}
\hline Fracturas \\
\hline Amputación traumática \\
\hline Luxaciones, esguinces y desgarros \\
\hline
\end{tabular}

Heridas

Q uemaduras

Envenenamientos

0 tros traumatismos

33674
786

2759

2341

1890

1709

21821

52161

Causas mal definidas

\begin{tabular}{rrr}
238 & 4.2 \\
\hline 4 & 123 & 2.2 \\
\hline 96 & 3 & 0.1 \\
\hline 1 & 10 & 0.2 \\
\hline 90 & 9 & 0.2 \\
\hline 9 & 7 & 0.1 \\
\hline 1 & 6 & 0.1 \\
\hline
\end{tabular}

* Incluye: Instituto Mexicano del Seguro Social, Instituto de Seguridad y Servicios Sociales de los Trabajadores del Estado y Secretaría de la D efensa Nacional

₹ Estimación realizada a partir de la muestra censal del Censo 2000 y las proyecciones de la población de México 2000-2030. Consejo Nacional de Población, 2002

Fuente: Secretaría de Salud. Dirección General de Información y Evaluación del Desempeño. Base de datos de Egresos Hospitalarios. México, 2002 


\section{Cuadro III \\ Grandes grupos de causas de morbilidad hospitalaria, población no derechohabiente.* Hombres, MÉxico, 2002}

Causas

Egresos

Tasa por $100000^{\ddagger}$

$\%$

Total

483372

1967

100.0

Enfermedades transmisibles, maternas, perinatales y nutricionales

\begin{tabular}{rrr}
114263 & 465 & 23.6 \\
\hline 29725 & 121 & 6.1 \\
\hline 33588 & 137 & 6.9 \\
\hline 47146 & 192 & 9.8 \\
\hline 3804 & 15 & 0.8
\end{tabular}

\begin{tabular}{|c|c|c|c|}
\hline Enfermedades no transmisibles & 257608 & 1048 & 53.3 \\
\hline Tumores malignos & 15181 & 62 & 3.1 \\
\hline 0 tros tumores & 6430 & 26 & 1.3 \\
\hline Diabetes mellitus & 15850 & 65 & 3.3 \\
\hline Enfermedades endócrinas, metabólicas, hematológicas e inmunológicas & 4868 & 20 & 1.0 \\
\hline Trastornos mentales y enfermedades del sistema nervioso central & 23409 & 95 & 4.8 \\
\hline Enfermedades de los órganos de los sentidos & 6818 & 28 & 1.4 \\
\hline Enfermedades cardiovasculares & 24910 & 101 & 5.2 \\
\hline Enfermedades respiratorias & 19738 & 80 & 4.1 \\
\hline Enfermedades digestivas & 72642 & 296 & 15.0 \\
\hline Enfermedades del sistema genito-urinario & 34710 & 141 & 7.2 \\
\hline Enfermedades de la piel & 11325 & 46 & 2.3 \\
\hline Enfermedades del sistema músculo-esquelético & 5897 & 24 & 1.2 \\
\hline Anomalías congénitas & 14320 & 58 & 3.0 \\
\hline Enfermedades de la boca & 1510 & 6 & 0.3 \\
\hline Lesiones & 93530 & 381 & 19.3 \\
\hline Fracturas & 35754 & 146 & 7.4 \\
\hline Amputación traumática & 1175 & 5 & 0.2 \\
\hline Luxaciones, esguinces y desgarros & 2844 & 12 & 0.6 \\
\hline Heridas & 12967 & 53 & 2.7 \\
\hline Q uemaduras & 4295 & 17 & 0.9 \\
\hline Envenenamientos & 5744 & 23 & 1.2 \\
\hline 0 tros traumatismos & 30751 & 125 & 6.4 \\
\hline
\end{tabular}

O tras causas de contacto con los servicios de salud

9576

Causas mal definidas

8395

$\begin{array}{ll}39 & 2.0 \\ 34 & 1.7\end{array}$

* Incluye: Secretaría de Salud (con Institutos N acionales de Salud y Hospital General de México) e IMSS-0 portunidades

‡ Estimación realizada a partir de la muestra censal del Censo 2000 y las proyecciones de la población de México 2000-2030. Consejo Nacional de Población, 2002

Fuente: Secretaría de Salud. Dirección General de Información y Evaluación del Desempeño. Base de datos de Egresos Hospitalarios. México, 2002 


\section{Cuadro IV \\ Grandes grupos de causas de morbilidad hospitalaria, población no derechohabiente.* Mujeres, México, 2002}

Causas

Total
Egresos

1362931

947571

Enfermedades transmisibles, maternas, perinatales y nutricionales

Enfermedades infecciosas y parasitarias

Infecciones respiratorias

Causas maternas

Ciertas afecciones originadas en el periodo perinatal

Deficiencias de la nutrición

Enfermedades no transmisibles

Tumores malignos

0 tros tumores

Diabetes mellitus

Enfermedades endócrinas, metabólicas, hematológicas e inmunológicas

Trastornos mentales y enfermedades del sistema nervioso central

Enfermedades de los órganos de los sentidos

Enfermedades cardiovasculares

Enfermedades respiratorias

Enfermedades digestivas

Enfermedades del sistema genito-urinario

Enfermedades de la piel

Enfermedades del sistema músculo-esquelético

A nomalías congénitas

Enfermedades de la boca

326007

23239

30246

21886

7159

15920

8088

27465

18919

87086

53989

11923

7029

11460

1598

\begin{tabular}{rrr}
947571 & 3869 & 69.5 \\
\hline 29871 & 122 & 2.2 \\
\hline 27558 & 113 & 2.0 \\
\hline 846842 & 3457 & 62.1 \\
38354 & 157 & 2.8 \\
4946 & 20 & 0.4
\end{tabular}

Tasa por $100000^{\ddagger}$

$\%$

5564

100.0

Lesiones $\quad 46919$

\begin{tabular}{|c|c|c|c|}
\hline Lesiones & 46919 & 192 & 3.4 \\
\hline Fracturas & 18634 & 76 & 1.4 \\
\hline Amputación traumática & 388 & 2 & 0.0 \\
\hline Luxaciones, esguinces y desgarros & 1518 & 6 & 0.1 \\
\hline Heridas & 3426 & 14 & 0.3 \\
\hline Q uemaduras & 2576 & 11 & 0.2 \\
\hline Envenenamientos & 4947 & 20 & 0.4 \\
\hline 0 tros traumatismos & 15430 & 63 & 1.1 \\
\hline 0 tras causas de contacto con los servicios de salud & 33764 & 138 & 2.5 \\
\hline Causas mal definidas & 8670 & 35 & 0.6 \\
\hline
\end{tabular}

Causas mal definidas

8670

$1331 \quad 23.9$

95

123

89

290.5

* Incluye: Secretaría de Salud (con Institutos N acionales de Salud y Hospital General de México) e IMSS-0 portunidades

₹ Estimación realizada a partir de la muestra censal del Censo 2000 y las proyecciones de la población de México 2000-2030. Consejo Nacional de Población, 2002

Fuente: Secretaría de Salud. Dirección General de Información y Evaluación del Desempeño. Base de datos de Egresos Hospitalarios. México, 2002 


\section{Cuadro V \\ MORBILIDAD HOSPITALARIA POR GRANDES GRUPOS DE CAUSA, SEGÚN INSTITUCIÓN. Hombres. Sector público del Sistema Nacional de Salud,* México, 2002}

\begin{tabular}{|c|c|c|c|c|c|c|c|c|c|c|c|}
\hline \multirow[b]{3}{*}{ Causas } & \multicolumn{11}{|c|}{ Institución } \\
\hline & Total & & SSA & & IMSS-0portu & nidades & IMSS & & ISSST & & Sedena \\
\hline & Egresos & $\%$ & Egresos & $\%$ & Egresos & $\%$ & Egresos & $\%$ & Egresos & $\%$ & Egresos \\
\hline tal & 131748710 & 100.0 & 440064 & 33.4 & 43308 & 3.3 & 681702 & 51.7 & 116101 & 8.8 & 36312 \\
\hline
\end{tabular}

Enfermedades transmisibles, maternas,

perinatales y nutricionales

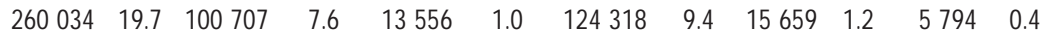

\begin{tabular}{lllllllllllll}
\hline Enfermedades infecciosas y parasitarias & 68767 & 5.2 & 26516 & 2.0 & 3209 & 0.2 & 30422 & 2.3 & 5239 & 0.4 & 3381 & 0.3 \\
\hline Infecciones respiratorias & 81410 & 6.2 & 29326 & 2.2 & 4262 & 0.3 & 40111 & 3.0 & 5625 & 0.4 & 2086 & 0.2 \\
\hline Ciertas afecciones originadas en el periodo & & & & & & & & & & &
\end{tabular}

Ciertas afecciones originadas en el periodo

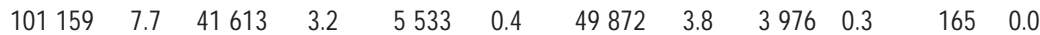

Deficiencias de la nutrición

$\begin{array}{llllllllllll}8698 & 0.7 & 3252 & 0.2 & 552 & 0.0 & 3913 & 0.3 & 819 & 0.1 & 162 & 0.0\end{array}$

Enfermedades no transmisibles

\begin{tabular}{lllllllllllll}
789 & 357 & 59.9 & 236211 & 17.9 & 21397 & 1.6 & 434094 & 32.9 & 79083 & 6.0 & 18572 & 1.4 \\
\hline
\end{tabular}

\begin{tabular}{|c|c|c|c|c|c|c|c|c|c|c|c|c|}
\hline Tumores malignos & 54153 & 4.1 & 14838 & 1.1 & 343 & 0.0 & 33459 & 2.5 & 4816 & 0.4 & 697 & 1 \\
\hline 0 tros tumores & 17921 & 1.4 & 5804 & 0.4 & 626 & 0.0 & 9359 & 0.7 & 1833 & 0.1 & 299 & \\
\hline Diabetes mellitus & 53714 & 4.1 & 13979 & 1.1 & 1871 & 0.1 & 30474 & 2.3 & 6287 & 0.5 & 1103 & \\
\hline $\begin{array}{l}\text { Enfermedades endócrinas, metabólicas, } \\
\text { hematológicas e inmunológicas }\end{array}$ & 14464 & 1.1 & 4591 & 0.3 & 277 & 0.0 & 8133 & 0.6 & 1210 & 0.1 & 253 & \\
\hline Trastornos mentales y enfermedades & & & & & & & & & & & & \\
\hline del sistema nervioso central & 43598 & 3.3 & 22323 & 1.7 & 1086 & 0.1 & 16846 & 1.3 & 2212 & 0.2 & 1131 & \\
\hline Enfermedades de los órganos de los sentidos & 20635 & 1.6 & 6632 & 0.5 & 186 & 0.0 & 9436 & 0.7 & 3162 & 0.2 & 1219 & \\
\hline Enfermedades cardiovasculares & 102358 & 7.8 & 22888 & 1.7 & 2022 & 0.2 & 63808 & 4.8 & 11476 & 0.9 & 2164 & \\
\hline Enfermedades respiratorias & 63514 & 4.8 & 17703 & 1.3 & 2035 & 0.2 & 35336 & 2.7 & 6788 & 0.5 & 1652 & \\
\hline Enfermedades digestivas & 192325 & 14.6 & 65000 & 4.9 & 7642 & 0.6 & 96874 & 7.4 & 19071 & 1.4 & 3738 & \\
\hline Enfermedades del sistema genito-urinario & 122558 & 9.3 & 31854 & 2.4 & 2856 & 0.2 & 71803 & 5.4 & 13294 & 1.0 & 2751 & \\
\hline Enfermedades de la piel & 44969 & 3.4 & 9972 & 0.8 & 1353 & 0.1 & 27129 & 2.1 & 4631 & 0.4 & 1884 & \\
\hline Enfermedades del sistema músculo-esquelético & 23307 & 1.8 & 5597 & 0.4 & 300 & 0.0 & 14276 & 1.1 & 2020 & 0.2 & 1114 & \\
\hline Anomalías congénitas & 32567 & 2.5 & 13628 & 1.0 & 692 & 0.1 & 15783 & 1.2 & 1992 & 0.2 & 472 & \\
\hline Enfermedades de la boca & 3274 & 0.2 & 1402 & 0.1 & 108 & 0.0 & 1378 & 0.1 & 291 & 0.0 & 95 & \\
\hline siones & 195136 & 14.8 & 87606 & 6.6 & 5924 & 0.4 & 85279 & 6.5 & 11823 & 0.9 & 4504 & \\
\hline Fracturas & 85341 & 6.5 & 35140 & 2.7 & 614 & 0.0 & 42184 & 3.2 & 5927 & 0.4 & 1476 & \\
\hline A mputación traumática & 3265 & 0.2 & 1059 & 0.1 & 116 & 0.0 & 1931 & 0.1 & 115 & 0.0 & 44 & \\
\hline Luxaciones, esguinces y desgarros & 7806 & 0.6 & 2752 & 0.2 & 92 & 0.0 & 3690 & 0.3 & 693 & 0.1 & 579 & \\
\hline Heridas & 19750 & 1.5 & 11667 & 0.9 & 1300 & 0.1 & 5744 & 0.4 & 667 & 0.1 & 372 & \\
\hline Q uemaduras & 8256 & 0.6 & 3918 & 0.3 & 377 & 0.0 & 3490 & 0.3 & 319 & 0.0 & 152 & \\
\hline Envenenamientos & 7540 & 0.6 & 4815 & 0.4 & 929 & 0.1 & 1484 & 0.1 & 219 & 0.0 & 93 & \\
\hline 0 tros traumatismos & 63178 & 4.8 & 28255 & 2.1 & 2496 & 0.2 & 26756 & 2.0 & 3883 & 0.3 & 1788 & \\
\hline
\end{tabular}

$\begin{array}{llllllllllllll}0 \text { tras causas de contacto con los servicios de salud } & 43458 & 3.3 & 8273 & 0.6 & 1303 & 0.1 & 21455 & 1.6 & 6300 & 0.5 & 6127 & 0.5\end{array}$

\begin{tabular}{lllllllllllll}
\hline Causas mal definidas & 29502 & 2.2 & 7267 & 0.6 & 1128 & 0.1 & 16556 & 1.3 & 3236 & 0.2 & 1315 & 0.1
\end{tabular}

* Incluye: Secretaría de Salud (SSA) (con Institutos N acionales de Salud y Hospital General de México) e IMSS-O portunidades, Instituto Mexicano del Seguro So cial (IMSS), Instituto de Seguridad y Servicios So ciales de los Trabajadores del Estado (ISSSTE) y Secretaría de la D efensa N acional (Sedena)

Fuente: Secretaría de Salud. Dirección General de Información y Evaluación del Desempeño. Base de datos de Egresos Hospitalarios. México, 2002 


\section{Cuadro VI \\ MoRbILIDAD HOSPITALARIA POR GRANDES GRUPOS DE CAUSA, SEGÚN INSTITUCIÓN. Mujeres. Sector público del Sistema Nacional de Salud,* México, 2002}

\begin{tabular}{|c|c|c|c|c|c|c|c|c|c|c|c|}
\hline \multirow[b]{3}{*}{ Causas } & \multicolumn{11}{|c|}{ Institución } \\
\hline & Tota & & SSA & & IM SS-Solid & ridads & IMSS & & ISSST & & Sedena \\
\hline & Egresos & $\%$ & Egresos & $\%$ & Egresos & $\%$ & Egresos & $\%$ & Egresos & $\%$ & Egresos \\
\hline otal & 2900228 & 100.0 & 1195237 & 41.2 & 167694 & 5.8 & 1266922 & 43.7 & 220907 & 7.6 & 49468 \\
\hline
\end{tabular}

Enfermedades transmisibles, maternas, perinatales

\begin{tabular}{|c|c|c|c|c|c|c|c|c|c|c|c|c|}
\hline utricionales & 1663924 & 57.4 & 829524 & 28.6 & 118047 & 4.1 & 617285 & 21.3 & 79229 & 2.7 & 19839 & 0.7 \\
\hline Enfermedades infecciosas y parasitarias & 74081 & 2.6 & 26050 & 0.9 & 3821 & 0.1 & 34618 & 1.2 & 7181 & 0.2 & 2411 & 0.1 \\
\hline Infecciones respiratorias & 66291 & 2.3 & 23982 & 0.8 & 3576 & 0.1 & 31822 & 1.1 & 5440 & 0.2 & 1471 & 0.1 \\
\hline Causas maternas & 1430170 & 49.3 & 741569 & 25.6 & 105273 & 3.6 & 505687 & 17.4 & 62080 & 2.1 & 15561 & 0.5 \\
\hline $\begin{array}{l}\text { Ciertas afecciones originadas en el periodo } \\
\text { perinatal }\end{array}$ & 81843 & 2.8 & 33778 & 1.2 & 4576 & 0.2 & 39993 & 1.4 & 3336 & 0.1 & 160 & 0.0 \\
\hline D eficiencias de la nutrición & 11539 & 0.4 & 4145 & 0.1 & 801 & 0.0 & 5165 & 0.2 & 1192 & 0.0 & 236 & 0.0 \\
\hline Enfermedades no transmisibles & 1007107 & 34.7 & 293893 & 10.1 & 32114 & 1.1 & 546947 & 18.9 & 114178 & 3.9 & 19975 & 0.7 \\
\hline Tumores malignos & 70587 & 2.4 & 22652 & 0.8 & 587 & 0.0 & 38936 & 1.3 & 7318 & 0.3 & 1094 & 0.0 \\
\hline 0 tros tumores & 89135 & 3.1 & 27286 & 0.9 & 2960 & 0.1 & 46947 & 1.6 & 11020 & 0.4 & 922 & 0.0 \\
\hline Diabetes mellitus & 66685 & 2.3 & 18712 & 0.6 & 3174 & 0.1 & 35047 & 1.2 & 7979 & 0.3 & 1773 & 0.1 \\
\hline $\begin{array}{l}\text { Enfermedades endócrinas, metabólicas, } \\
\text { hematológicas e inmunológicas }\end{array}$ & 21284 & 0.7 & 6719 & 0.2 & 440 & 0.0 & 11545 & 0.4 & 2236 & 0.1 & 344 & 0.0 \\
\hline $\begin{array}{l}\text { Trastornos mentales y enfermedades } \\
\text { del sistema nervioso central }\end{array}$ & 38150 & 1.3 & 15080 & 0.5 & 840 & 0.0 & 18291 & 0.6 & 2967 & 0.1 & 972 & 0.0 \\
\hline Enfermedades de los órganos de los s & 23478 & 0.8 & 7900 & 0.3 & 188 & 0.0 & 10140 & 0.3 & 4238 & 0.1 & 1012 & 0.0 \\
\hline Enfermedades cardiovasculares & 106760 & 3.7 & 24734 & 0.9 & 2731 & 0.1 & 63911 & 2.2 & 13111 & 0.5 & 2273 & 0.1 \\
\hline Enfermedades respiratorias & 59049 & 2.0 & 16706 & 0.6 & 2213 & 0.1 & 31674 & 1.1 & 7084 & 0.2 & 1372 & 0.0 \\
\hline Enfermedades digestivas & 243987 & 8.4 & 76777 & 2.6 & 10309 & 0.4 & 126621 & 4.4 & 25650 & 0.9 & 4630 & 0.2 \\
\hline Enfermedades del sistema genito-urinario & 182769 & 6.3 & 47938 & 1.7 & 6051 & 0.2 & 105459 & 3.6 & 20339 & 0.7 & 2982 & 0.1 \\
\hline Enfermedades de la piel & 47556 & 1.6 & 10481 & 0.4 & 1442 & 0.0 & 27261 & 0.9 & \multicolumn{2}{|c|}{69440.2} & 1428 & 0.0 \\
\hline Enfermedades del sistema músculo-esquelético & 26725 & 0.9 & 6493 & 0.2 & 536 & 0.0 & 15870 & 0.5 & \multicolumn{2}{|c|}{31340.1} & 692 & 0.0 \\
\hline Anomalías congénitas & 27342 & 0.9 & 10949 & 0.4 & 511 & 0.0 & 13698 & 0.5 & \multicolumn{2}{|c|}{17810.1} & 403 & 0.0 \\
\hline Enfermedades de la boca & 3600 & 0.1 & 1466 & 0.1 & 132 & 0.0 & 1547 & 0.1 & \multicolumn{2}{|c|}{3770.0} & 78 & 0.0 \\
\hline Lesiones & 111899 & 3.9 & 43375 & 1.5 & 3544 & 0.1 & 52057 & 1.8 & \multicolumn{2}{|c|}{109880.4} & 1935 & 0.1 \\
\hline Fracturas & 52308 & 1.8 & 18311 & 0.6 & 323 & 0.0 & 27066 & 0.9 & \multicolumn{2}{|c|}{58940.2} & 714 & 0.0 \\
\hline Amputación traumática & 1174 & 0.0 & 347 & 0.0 & 41 & 0.0 & 705 & 0.0 & \multicolumn{2}{|c|}{690.0} & 12 & 0.0 \\
\hline Luxaciones, esguinces y desgarros & 4277 & 0.1 & 1467 & 0.1 & 51 & 0.0 & 1938 & 0.1 & \multicolumn{2}{|c|}{6470.0} & 174 & 0.0 \\
\hline Heridas & 5767 & 0.2 & 3014 & 0.1 & 412 & 0.0 & 1827 & 0.1 & \multicolumn{2}{|c|}{3970.0} & 117 & 0.0 \\
\hline Q uemaduras & 4466 & 0.2 & 2294 & 0.1 & 282 & 0.0 & 1608 & 0.1 & \multicolumn{2}{|c|}{2120.0} & 70 & 0.0 \\
\hline Envenenamientos & 6656 & 0.2 & 4129 & 0.1 & 818 & 0.0 & 1347 & 0.0 & \multicolumn{2}{|c|}{2900.0} & 72 & 0.0 \\
\hline 0 tros traumatismos & 37251 & 1.3 & 13813 & 0.5 & 1617 & 0.1 & 17566 & 0.6 & \multicolumn{2}{|c|}{34790.1} & 776 & 0.0 \\
\hline 0 tras causas de contacto con los servicios de salud & d 85925 & 3.0 & 20990 & 0.7 & 12774 & 0.4 & 33483 & 1.2 & \multicolumn{2}{|c|}{121960.4} & 6482 & 0.2 \\
\hline Causas mal definidas & 31373 & 1.1 & 7455 & 0.3 & 1215 & 0.0 & 17150 & 0.6 & \multicolumn{2}{|c|}{43160.1} & 1237 & 0.0 \\
\hline
\end{tabular}

* Incluye: Secretaría de Salud (SSA) (con Institutos N acionales de Salud y Hospital General de México) e IMSS-O portunidades, Instituto Mexicano del Seguro Social (IMSS), Instituto de Seguridad y Servicios Sociales de los Trabajadores del Estado (ISSSTE) y Secretaría de la D efensa N acional (Sedena)

Fuente: Secretaría de Salud. Dirección General de Información y Evaluación del Desempeño. Base de datos de Egresos Hospitalarios. México, 2002 


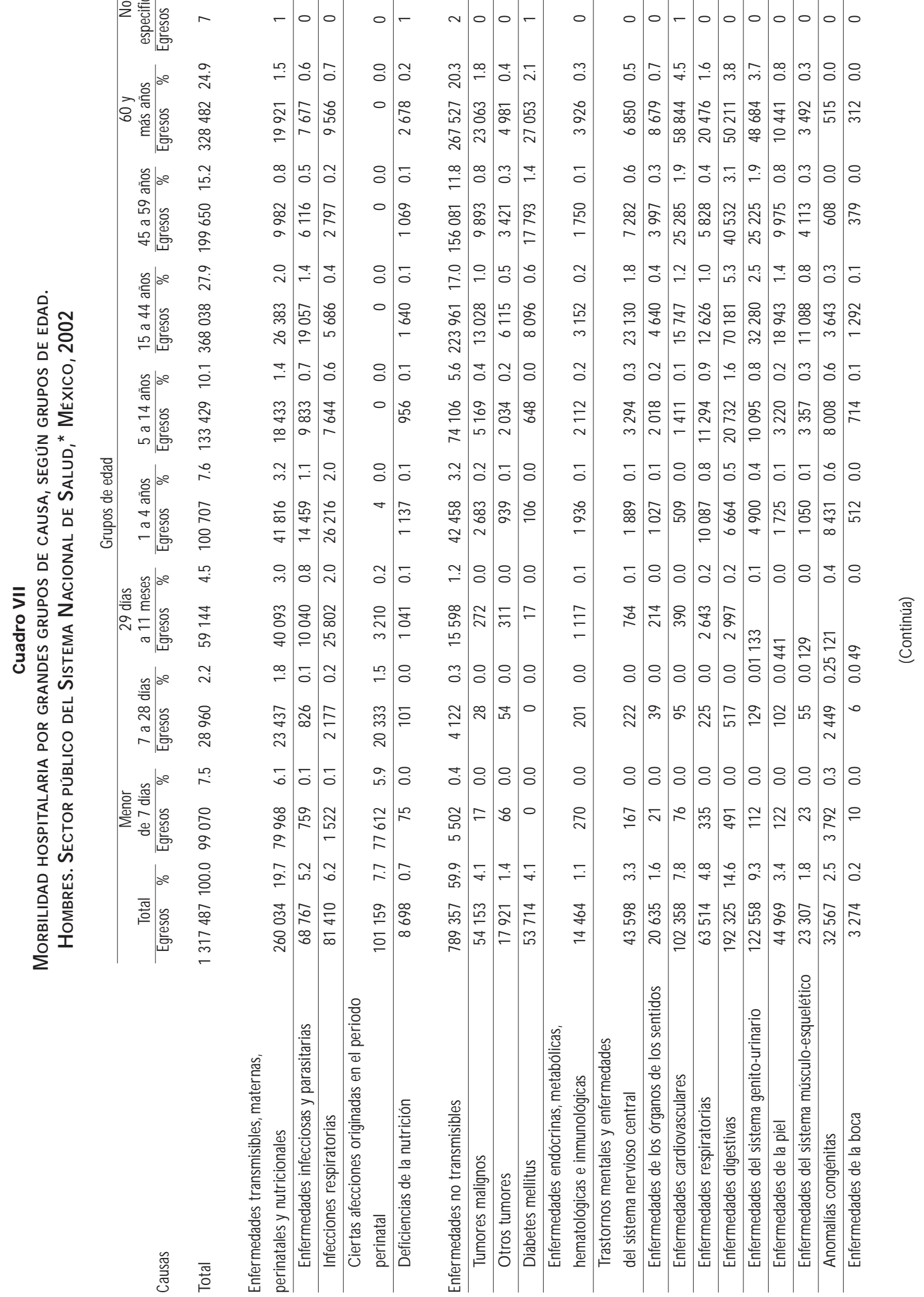

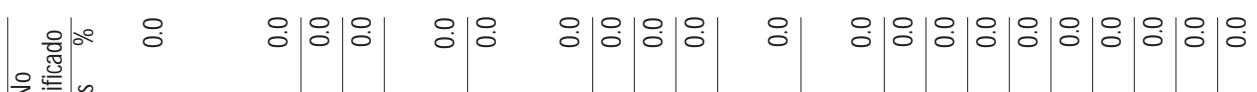




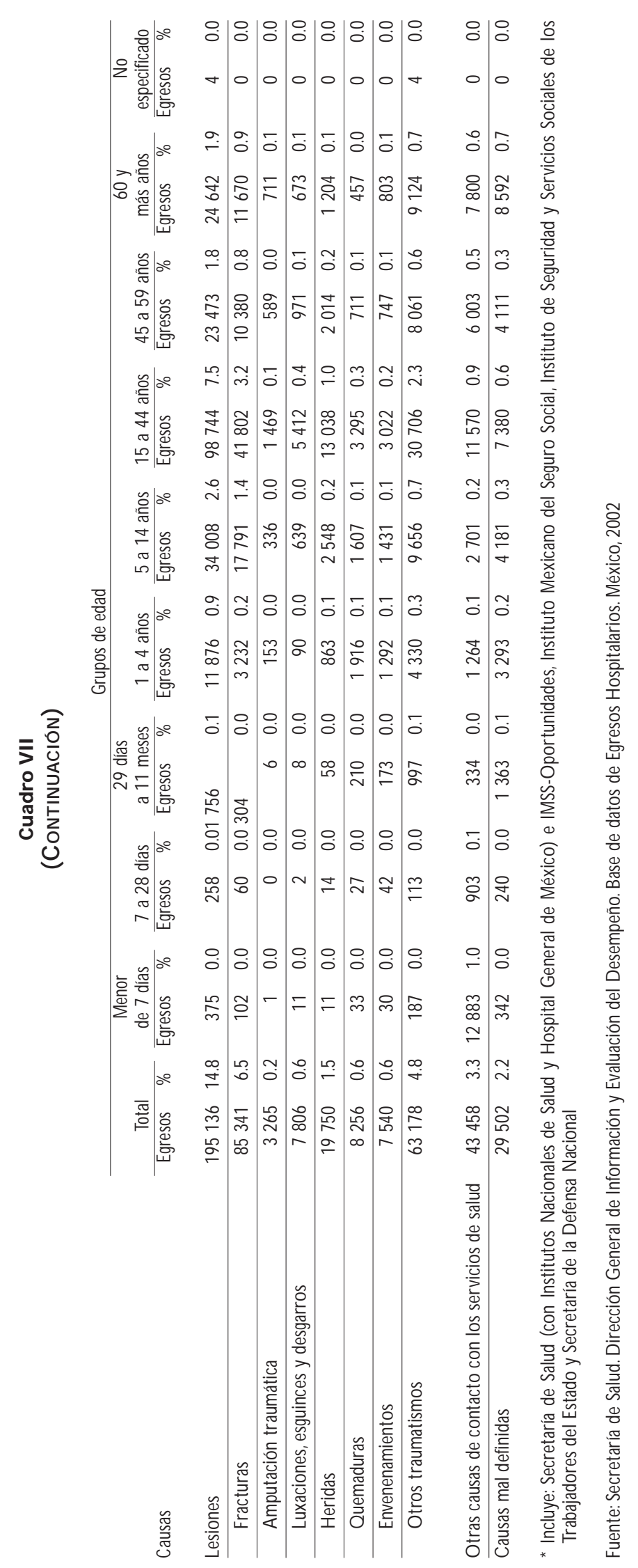




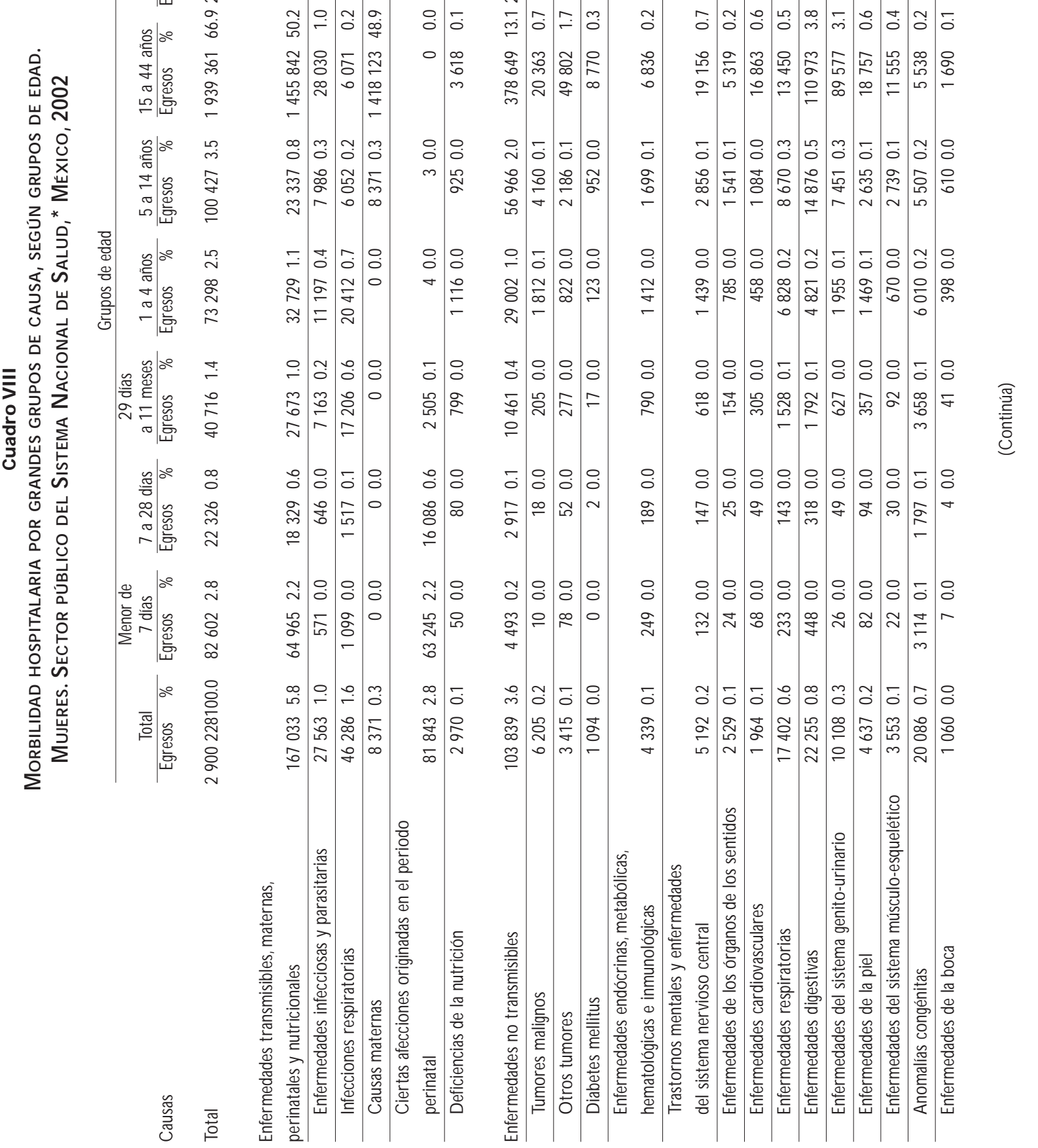

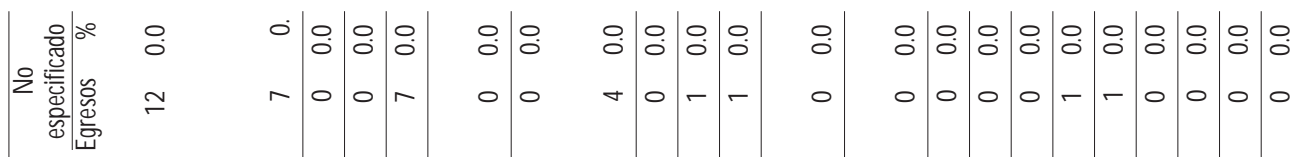

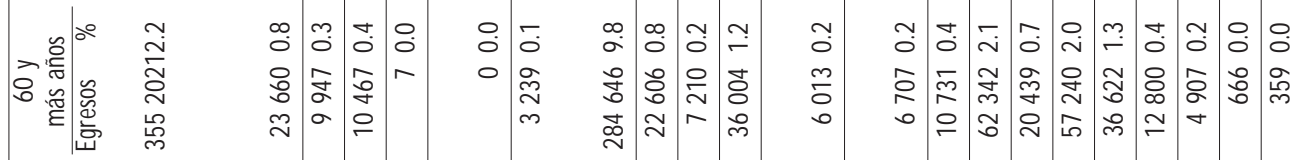
秃

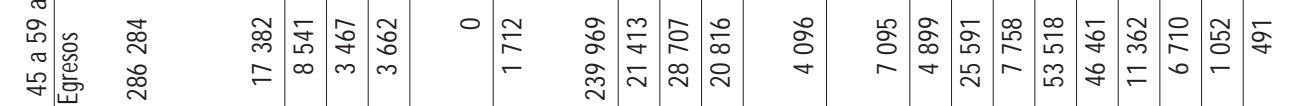




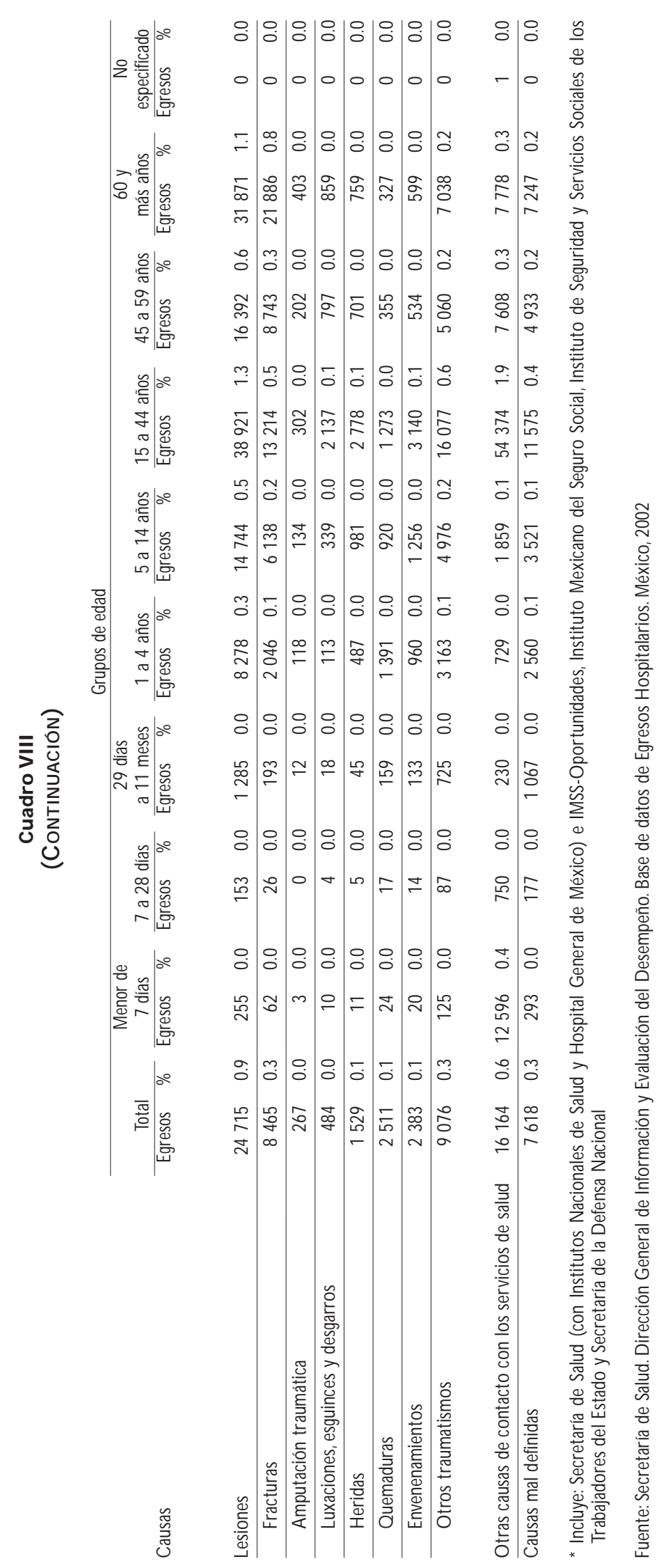




\section{Cuadro IX \\ Grandes GRUPOS DE CAUSAS DE MORTALIDAD hOSPITALARIA, Población derechohabiente.* Hombres, México, 2002}

Causas

Defunciones

Tasa por $100000^{\ddagger}$

$\%$

Total

34878

131

100.0

Enfermedades transmisibles, maternas, perinatales y nutricionales

\begin{tabular}{rrr}
6040 & 23 & 17.3 \\
\hline 1916 & 7 & 5.5 \\
\hline 1463 & 5 & 4.2 \\
\hline 2335 & 9 & 6.7 \\
\hline 326 & 1 & 0.9
\end{tabular}

\begin{tabular}{|c|c|c|c|}
\hline Enfermedades no transmisibles & 27235 & 102 & 78.1 \\
\hline Tumores malignos & 4521 & 17 & 13.0 \\
\hline 0 tros tumores & 250 & 1 & 0.7 \\
\hline Diabetes mellitus & 4539 & 17 & 13.0 \\
\hline Enfermedades endócrinas, metabólicas, hematológicas e inmunológicas & 406 & 2 & 1.2 \\
\hline Trastornos mentales y enfermedades del sistema nervioso central & 572 & 2 & 1.6 \\
\hline Enfermedades de los órganos de los sentidos & 1 & 0 & 0.0 \\
\hline Enfermedades cardiovasculares & 7238 & 27 & 20.8 \\
\hline Enfermedades respiratorias & 2175 & 8 & 6.2 \\
\hline Enfermedades digestivas & 4419 & 17 & 12.7 \\
\hline Enfermedades del sistema genito-urinario & 1873 & 7 & 5.4 \\
\hline Enfermedades de la piel & 143 & 1 & 0.4 \\
\hline Enfermedades del sistema músculo-esquelético & 65 & 0 & 0.2 \\
\hline Anomalías congénitas & 1021 & 4 & 2.9 \\
\hline Enfermedades de la boca & 12 & 0 & 0.0 \\
\hline
\end{tabular}

\begin{tabular}{lrrr} 
Lesiones & 1082 & 4 & 3.1 \\
\hline Accidentes de tránsito & 330 & 1 & 0.9 \\
\hline Caídas accidentales & 329 & 1 & 0.9 \\
\hline Envenenamiento accidental & 7 & 0 & 0.0 \\
\hline Exposiciones al fuego, humo y llamas & 35 & 0 & 0.1 \\
\hline Otros accidentes & 305 & 1 & 0.9 \\
\hline Suicidios & 22 & 0 & 0.1 \\
\hline Homicidios & 54 & 0 & 0.2
\end{tabular}

0 tras lesiones intencionales

Causas mal definidas

$\begin{array}{rrr}33 & 0 & 0.1 \\ 488 & 2 & 1.4\end{array}$

* Incluye: Instituto Mexicano del Seguro Social, Instituto de Seguridad y Servicios Sociales de los Trabajadores del Estado y Secretaría de la Defensa Nacional

‡ Estimación realizada a partir de la muestra censal del Censo 2000 y las proyecciones de la población de México 2000-2030. Consejo Nacional de Población, 2002

Fuente: Secretaría de Salud. Dirección General de Información y Evaluación del Desempeño. Base de datos de Egresos Hospitalarios. México, 2002 


\section{Cuadro $X$}

\section{Grandes GRUPos de CAUSAS DE MORTALIDAD HOSPITALARIA.} Población derechohabiente.* Mujeres, México, 2002

Causas

Total
Defunciones

33578

4915

Enfermedades transmisibles, maternas, perinatales y nutricionales

Enfermedades infecciosas y parasitarias

Infecciones respiratorias

Causas maternas

Ciertas afecciones originadas en el periodo perinatal

Deficiencias de la nutrición

Enfermedades no transmisibles

\begin{tabular}{lr} 
Enfermedades no transmisibles & 277 \\
\hline Tumores malignos & 4652 \\
\hline O tros tumores & 314 \\
\hline Diabetes mellitus & 458 \\
\hline Enfermedades endócrinas, metabólicas, hematológicas e inmunológicas & 530 \\
\hline Trastornos mentales y enfermedades del sistema nervioso central & 8062 \\
\hline Enfermedades de los órganos de los sentidos & 1897 \\
\hline Enfermedades cardiovasculares & 3449 \\
\hline Enfermedades respiratorias & 1580 \\
\hline Enfermedades digestivas & 200 \\
\hline Enfermedades del sistema genito-urinario & 238 \\
\hline Enfermedades de la piel & 793 \\
\hline Enfermedades del sistema músculo-esquelético &
\end{tabular}

Tasa por $100000^{\ddagger} \quad \%$

123

100.0

\begin{tabular}{rrr}
1279 & 5 & 3.8 \\
1381 & 5 & 4.1 \\
135 & 0 & 0.4 \\
1790 & 7 & 5.3 \\
\hline 330 & 1 & 1.0
\end{tabular}

$\begin{array}{lll}27718 & 102 & 82.5\end{array}$

$\begin{array}{lll}4652 & 17 & 13.9\end{array}$

$314 \quad 1 \quad 0.9$

$\begin{array}{lll}538 & 20 & 16.5\end{array}$

$\begin{array}{lll}459 & 2 & 1.4\end{array}$

$\begin{array}{lll}530 & 2 & 1.6\end{array}$

$\begin{array}{lll}2 & 0 & 0.0\end{array}$

$062 \quad 30 \quad 24.0$

$\begin{array}{lll}897 & 7 & 5.6\end{array}$

$\begin{array}{lll}449 & 13 & 10.3\end{array}$

$\begin{array}{lll}580 & 6 & 4.7\end{array}$

$\begin{array}{lll}200 & 1 & 0.6\end{array}$

$\begin{array}{lll}238 & 1 & 0.7\end{array}$

\begin{tabular}{rll}
793 & 1 & 0.7 \\
4 & 0 & 2.4 \\
\hline
\end{tabular}

\begin{tabular}{lccc} 
Lesiones & 512 & 2 & 1.5 \\
\hline Accidentes de tránsito & 87 & 0 & 0.3 \\
\hline Caídas accidentales & 256 & 1 & 0.8 \\
\hline Envenenamiento accidental & 6 & 0 & 0.0 \\
\hline Exposiciones al fuego, humo y llamas & 14 & 0 & 0.0 \\
\hline Otros accidentes & 126 & 0 & 0.4 \\
\hline Suicidios & 14 & 0 & 0.0 \\
\hline Homicidios & 9 & 0 & 0.0
\end{tabular}

0 tras lesiones intencionales

$\begin{array}{rrr}19 & 0 & 0.1 \\ 414 & 2 & 1.2\end{array}$

* Incluye: Instituto Mexicano del Seguro Social, Instituto de Seguridad y Servicios Sociales de los Trabajadores del Estado y Secretaría de la Defensa Nacional

‡ Estimación realizada a partir de la muestra censal del Censo 2000 y las proyecciones de la población de México 2000-2030. Consejo Nacional de Población, 2002

Fuente: Secretaría de Salud. Dirección General de Información y Evaluación del Desempeño. Base de datos de Egresos Hospitalarios. México, 2002 


\section{Cuadro XI \\ Grandes GRUPOS de CAUSAS DE MORTALIDAD hospitalaria. Población no derechohabiente.* Hombres, México, 2002}

Causas

Defunciones

Tasa por $100000^{\ddagger}$

$\%$

Total

23823

97

100.0

Enfermedades transmisibles, maternas, perinatales y nutricionales

\begin{tabular}{rrr}
8387 & 34 & 35.2 \\
\hline 2191 & 9 & 9.2 \\
\hline 1134 & 5 & 4.8 \\
\hline 4718 & 19 & 19.8 \\
\hline 344 & 1 & 1.4
\end{tabular}

Ciertas afecciones originadas en el periodo perinatal

Deficiencias de la nutrición

\begin{tabular}{rrr}
13548 & 55 & 56.9 \\
1401 & 6 & 5.9 \\
147 & 1 & 0.6 \\
\hline 1575 & 6 & 6.6 \\
\hline 399 & 2 & 1.7 \\
\hline 792 & 3 & 3.3 \\
\hline 1 & 0 & 0.0 \\
\hline 3062 & 12 & 12.9 \\
\hline 1163 & 5 & 4.9 \\
\hline 3165 & 13 & 13.3 \\
\hline 790 & 3 & 3.3 \\
\hline 34 & 0 & 0.1 \\
\hline 30 & 0 & 0.1 \\
\hline 983 & 4 & 4.1 \\
6 & 0 & 0.0
\end{tabular}

\begin{tabular}{l} 
Lesiones \\
\hline Accidentes de tránsito \\
\hline Caídas accidentales \\
\hline Envenenamiento accidental \\
\hline Exposiciones al fuego, humo y llamas \\
\hline O tros accidentes \\
\hline Suicidios \\
\hline Homicidios
\end{tabular}

0 tras lesiones intencionales

\begin{tabular}{rll}
1436 & 6 & 6.0 \\
\hline 459 & 2 & 1.9 \\
\hline 241 & 1 & 1.0 \\
\hline 37 & 0 & 0.2 \\
\hline 32 & 0 & 0.1 \\
\hline 473 & 2 & 2.0 \\
\hline 43 & 0 & 0.2 \\
\hline 151 & 1 & 0.6
\end{tabular}

Causas mal definidas

106

$\begin{array}{ll}0 & 0.4 \\ 1 & 1.5\end{array}$

* Incluye: Secretaría de Salud (con Institutos N acionales de Salud y Hospital General de México) e IMSS-0 portunidades

₹ Estimación realizada a partir de la muestra censal del Censo 2000 y las proyecciones de la población de México 2000-2030. Consejo Nacional de Población, 2002

Fuente: Secretaría de Salud. Dirección General de Información y Evaluación del Desempeño. Base de datos de Egresos Hospitalarios. México, 2002 


\section{Cuadro XII \\ Grandes Grupos de CAUSAS DE MORTALIDAD hospitalaria. Población no derechohabiente.* Mujeres, México, 2002}

Causas

Total
Defunciones

18969
Tasa por $100000^{\ddagger}$

77

\begin{tabular}{rrr}
6311 & 26 & 33.3 \\
\hline 1374 & 6 & 7.2 \\
\hline 972 & 4 & 5.1 \\
\hline 3354 & 1 & 1.5 \\
\hline 318 & 14 & 17.7 \\
\hline
\end{tabular}

Enfermedades no transmisibles

\begin{tabular}{rrr}
11935 & 49 & 62.9 \\
\hline 1507 & 6 & 7.9 \\
157 & 1 & 0.8 \\
1942 & 8 & 10.2 \\
\hline 418 & 2 & 2.2 \\
\hline 332 & 1 & 1.8 \\
\hline 1 & 0 & 0.0 \\
\hline 332 & 14 & 17.6 \\
\hline 977 & 4 & 5.2 \\
\hline 1661 & 7 & 8.8 \\
\hline 623 & 3 & 3.3 \\
\hline 58 & 0 & 0.3 \\
\hline 95 & 0 & 0.5 \\
\hline 829 & 3 & 4.4 \\
\hline 3 & 0 & 0.0
\end{tabular}

Lesiones

Tumores malignos

0 tros tumores

Diabetes mellitus

Enfermedades endócrinas, metabólicas, hematológicas e inmunológicas

Trastornos mentales y enfermedades del sistema nervioso central

Enfermedades de los órganos de los sentidos

Enfermedades cardiovasculares

Enfermedades respiratorias

Enfermedades digestivas

Enfermedades del sistema genito-urinario

Enfermedades de la piel

Enfermedades del sistema músculo-esquelético

Anomalías congénitas

Enfermedades de la boca

\begin{tabular}{rrr}
456 & 2 & 2.4 \\
124 & 1 & 0.7 \\
89 & 0 & 0.5 \\
15 & 0 & 0.1 \\
17 & 0 & 0.1 \\
153 & 1 & 0.8 \\
30 & 0 & 0.2 \\
\hline 28 & 0 & 0.1
\end{tabular}

0 tras lesiones intencionales

22

245

$\%$

100.0

Accidentes de tránsito
Caídas accidentales
Envenenamiento accidental
Exposiciones al fuego, humo y llamas
Otros accidentes
Suicidios
Homicidios

Causas mal definidas

* Incluye: Secretaría de Salud (con Institutos N acionales de Salud y Hospital General de México) e IMSS-0 portunidades

₹ Estimación realizada a partir de la muestra censal del Censo 2000 y las proyecciones de la población de México 2000-2030. Consejo Nacional de Población, 2002

Fuente: Secretaría de Salud. Dirección General de Información y Evaluación del Desempeño. Base de datos de Egresos Hospitalarios. México, 2002 


\section{Cuadro XIII \\ MORTALIDAD HOSPITALARIA POR GRANDES GRUPOS DE CAUSA, SEGÚN INSTITUCIÓN. Hombres. Sector público del Sistema Nacional de Salud,* México, 2002}

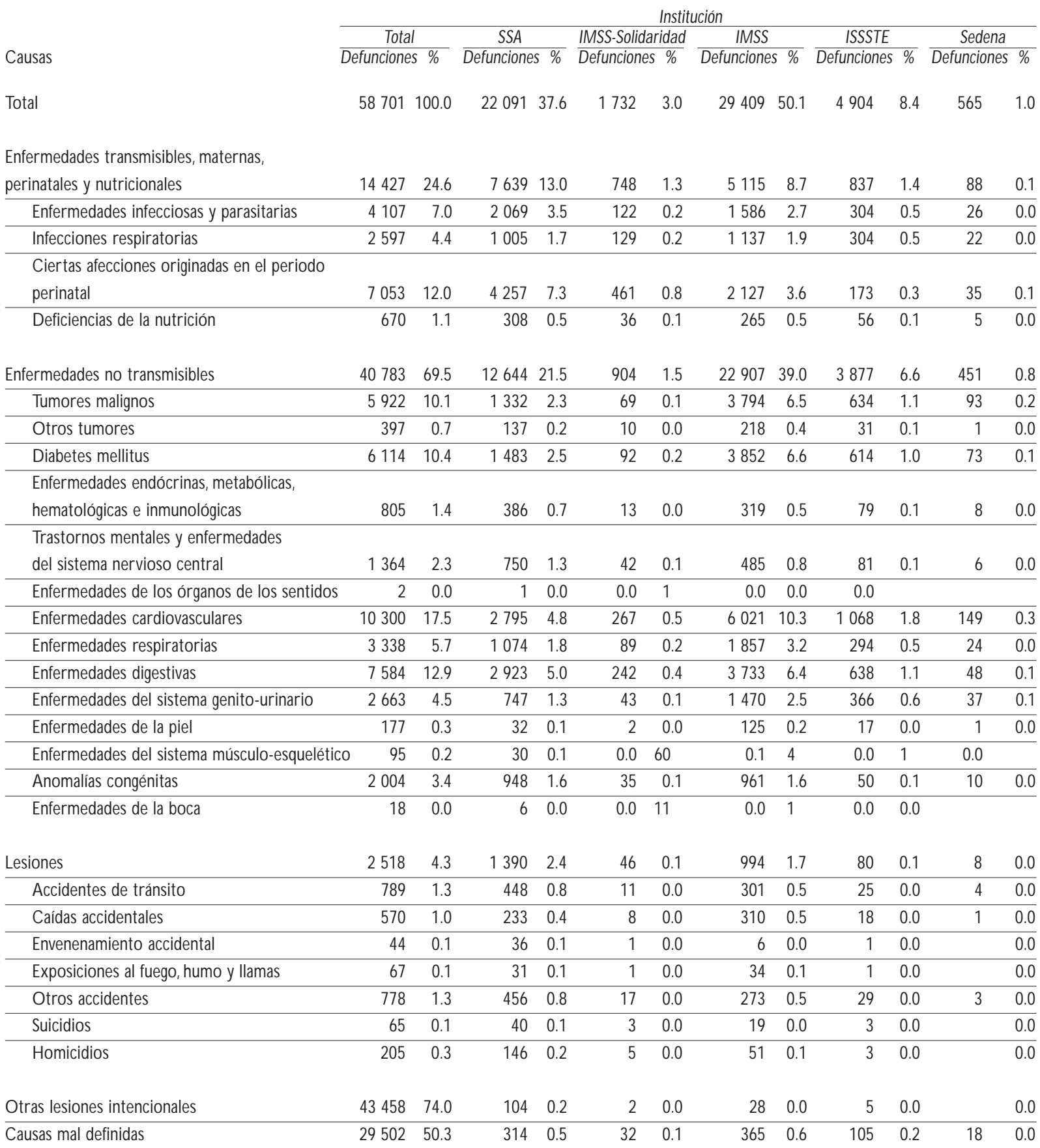

* Incluye: Secretaría de Salud (SSA) (con Institutos N acionales de Salud y Hospital General de México) e IMSS-0 portunidades, Instituto Mexicano del Seguro Social (IMSS), Instituto de Seguridad y Servicios Sociales de los Trabajadores del Estado (ISSSTE) y Secretaría de la D efensa N acional (Sedena)

Fuente: Secretaría de Salud. Dirección General de Información y Evaluación del Desempeño. Base de datos de Egresos Hospitalarios. México, 2002 


\section{Cuadro XIV \\ Mortalidad hospitalaria POR GRANDES GRUPOS DE CAUSA, SEGÚN INSTITUCIÓN. Mujeres. Sector público del Sistema Nacional de Salud,* México, 2002}

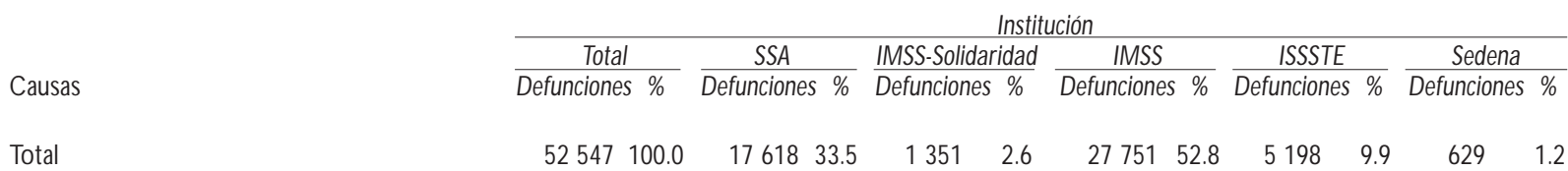

Enfermedades transmisibles, maternas,

perinatales y nutricionales

\begin{tabular}{llllllllllll}
11226 & 21.4 & 5757 & 11.0 & 554 & 1.1 & 4085 & 7.8 & 732 & 1.4 & 98 & 0.2 \\
\hline
\end{tabular}

\begin{tabular}{|c|c|c|c|c|c|c|c|c|c|c|c|c|}
\hline Enfermedades infecciosas y parasitarias & 2653 & 5.0 & 1283 & 2.4 & 91 & 0.2 & 1032 & 2.0 & 228 & 0.4 & 19 & 0.0 \\
\hline Infecciones respiratorias & 2353 & 4.5 & 858 & 1.6 & 114 & 0.2 & 1023 & 1.9 & 326 & 0.6 & 32 & 0.1 \\
\hline Causas maternas & 429 & 0.8 & 268 & 0.5 & 26 & 0.0 & 113 & 0.2 & 20 & 0.0 & 2 & 0.0 \\
\hline \multicolumn{13}{|l|}{ Ciertas afecciones originadas en el periodo } \\
\hline perinatal & 5143 & 9.8 & 3064 & 5.8 & 289 & 0.5 & 1647 & 3.1 & 109 & 0.2 & 34 & 0.1 \\
\hline D eficiencias de la nutrición & 648 & 1.2 & 284 & 0.5 & 34 & 0.1 & 270 & 0.5 & 49 & 0.1 & 11 & 0.0 \\
\hline
\end{tabular}

Enfermedades no transmisibles

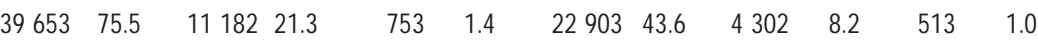

\begin{tabular}{|c|c|c|c|c|c|c|c|c|c|c|c|c|}
\hline Tumores malignos & 6159 & 11.7 & 1432 & 2.7 & 75 & 0.1 & 3809 & 7.2 & 741 & 1.4 & 102 & 0.2 \\
\hline 0 tros tumores & 471 & 0.9 & 144 & 0.3 & 13 & 0.0 & 258 & 0.5 & 53 & 0.1 & 3 & 0.0 \\
\hline Diabetes mellitus & 7480 & 14.2 & 1804 & 3.4 & 138 & 0.3 & 4682 & 8.9 & 754 & 1.4 & 102 & 0.2 \\
\hline $\begin{array}{l}\text { Enfermedades endócrinas, metabólicas, } \\
\text { hematológicas e inmunológicas }\end{array}$ & 877 & 1.7 & 404 & 0.8 & 14 & 0.0 & 338 & 0.6 & 111 & 0.2 & 10 & 0.0 \\
\hline $\begin{array}{l}\text { Trastornos mentales y enfermedades } \\
\text { del sistema nervioso central }\end{array}$ & 862 & 1.6 & 325 & 0.6 & 7 & 0.0 & 435 & 0.8 & 89 & 0.2 & 6 & 0.0 \\
\hline Enfermedades de los órganos de los sentidos & 3 & 0.0 & 1 & 0.0 & 0.0 & 1 & 0.0 & 1 & 0.0 & 0.0 & & \\
\hline Enfermedades cardiovasculares & 11394 & 21.7 & 3102 & 5.9 & 230 & 0.4 & 6573 & 12.5 & 1329 & 2.5 & 160 & 0.3 \\
\hline Enfermedades respiratorias & 2874 & 5.5 & 889 & 1.7 & 88 & 0.2 & 1543 & 2.9 & 330 & 0.6 & 24 & 0.0 \\
\hline Enfermedades digestivas & 5110 & 9.7 & 1548 & 2.9 & 113 & 0.2 & 2922 & 5.6 & 467 & 0.9 & 60 & 0.1 \\
\hline Enfermedades del sistema genito-urinario & 2203 & 4.2 & 596 & 1.1 & 27 & 0.1 & 1207 & 2.3 & 339 & 0.6 & 34 & 0.1 \\
\hline Enfermedades de la piel & 258 & 0.5 & 51 & 0.1 & 7 & 0.0 & 178 & 0.3 & 21 & 0.0 & 1 & 0.0 \\
\hline Enfermedades del sistema músculo-esquelético & 333 & 0.6 & 95 & 0.2 & \multicolumn{2}{|c|}{0.0210} & 0.4 & 25 & 0.0 & 3 & 0.0 & \\
\hline Anomalías congénitas & 1622 & 3.1 & 788 & 1.5 & 41 & 0.1 & 744 & 1.4 & 41 & 0.1 & 8 & 0.0 \\
\hline Enfermedades de la boca & 7 & 0.0 & 3 & 0.0 & 0.0 & 3 & 0.0 & 1 & 0.0 & 0.0 & & \\
\hline Lesiones & 968 & 1.8 & 444 & 0.8 & 12 & 0.0 & 446 & 0.8 & 65 & 0.1 & 1 & 0.0 \\
\hline Accidentes de tránsito & 211 & 0.4 & 122 & 0.2 & 2 & 0.0 & 76 & 0.1 & 11 & 0.0 & 0.0 & \\
\hline Caídas accidentales & 345 & 0.7 & 88 & 0.2 & 1 & 0.0 & 231 & 0.4 & 24 & 0.0 & 1 & 0.0 \\
\hline Envenenamiento accidental & 21 & 0.0 & 14 & 0.0 & 1 & 0.0 & 5 & 0.0 & 1 & 0.0 & 0.0 & \\
\hline Exposiciones al fuego, humo y llamas & 31 & 0.1 & 17 & 0.0 & 0.0 & 14 & 0.0 & 0.0 & 0.0 & & & \\
\hline 0 tros accidentes & 279 & 0.5 & 147 & 0.3 & 6 & 0.0 & 104 & 0.2 & 22 & 0.0 & 0.0 & \\
\hline Suicidios & 44 & 0.1 & 29 & 0.1 & 1 & 0.0 & 9 & 0.0 & 5 & 0.0 & 0.0 & \\
\hline Homicidios & 37 & 0.1 & 27 & 0.1 & 1 & 0.0 & 7 & 0.0 & 2 & 0.0 & 0.0 & \\
\hline
\end{tabular}

0 tras lesiones intencionales

$\begin{array}{rrrrrrrrrrrr}41 & 0.1 & 21 & 0.0 & 1 & 0.0 & 10 & 0.0 & 9 & 0.0 & 0.0 & \\ 659 & 1.3 & 214 & 0.4 & 31 & 0.1 & 307 & 0.6 & 90 & 0.2 & 17 & 0.0\end{array}$

Causas mal definidas

* Incluye: Secretaría de Salud (SSA) (con Institutos N acionales de Salud y Hospital General de México) e IMSS-O portunidades, Instituto Mexicano del Seguro Social (IMSS), Instituto de Seguridad y Servicios Sociales de los Trabajadores del Estado (ISSSTE) y Secretaría de la D efensa N aciona (Sedena)

Fuente: Secretaría de Salud. Dirección General de Información y Evaluación del Desempeño. Base de datos de Egresos Hospitalarios. México, 2002 
|

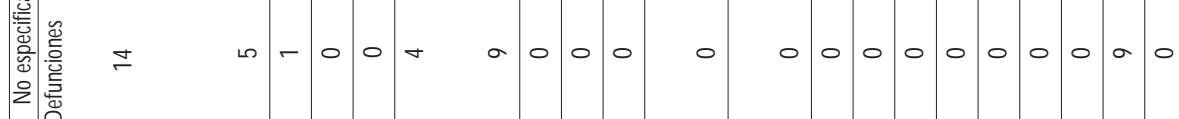

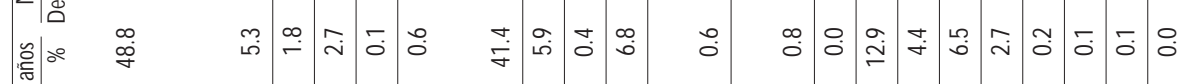

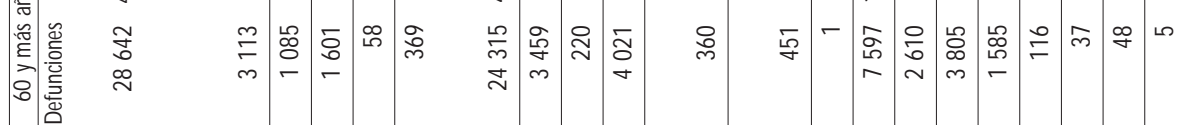

皇

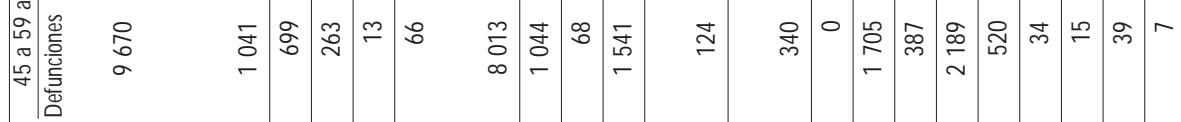

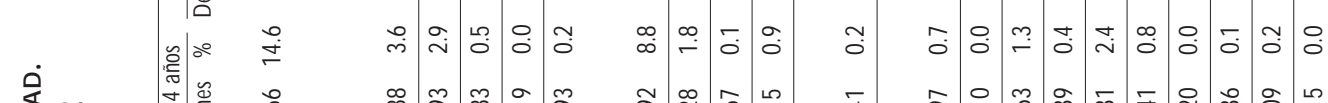

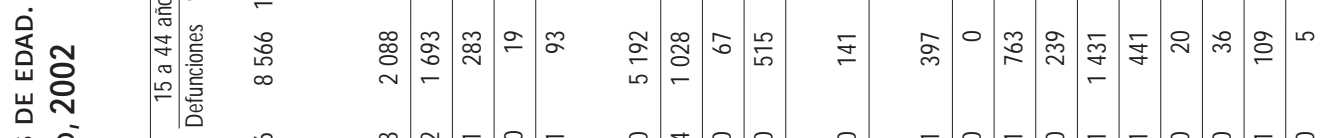

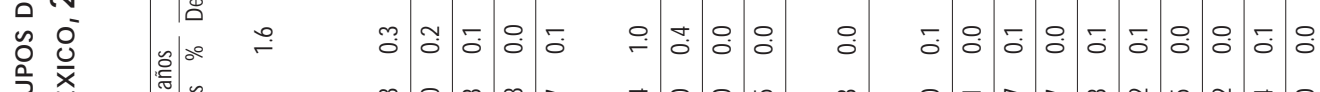

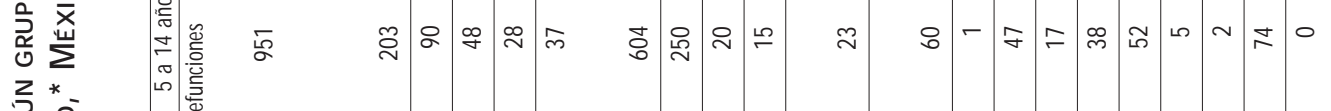

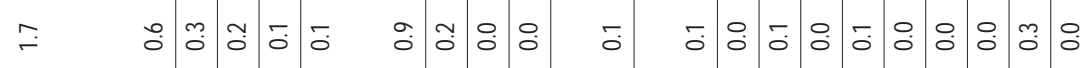

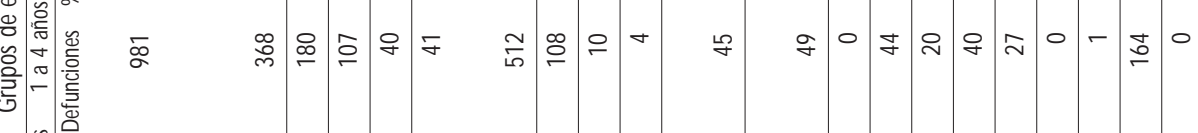

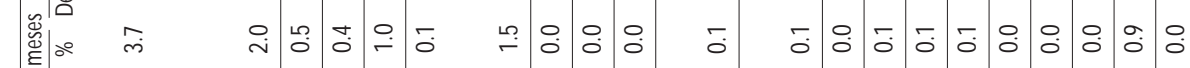

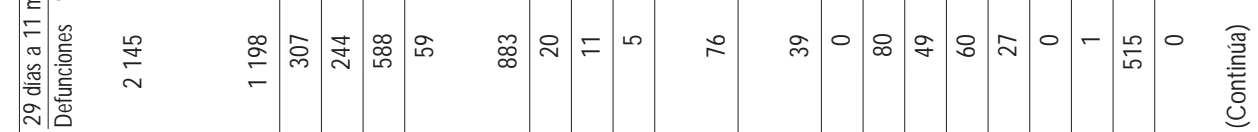

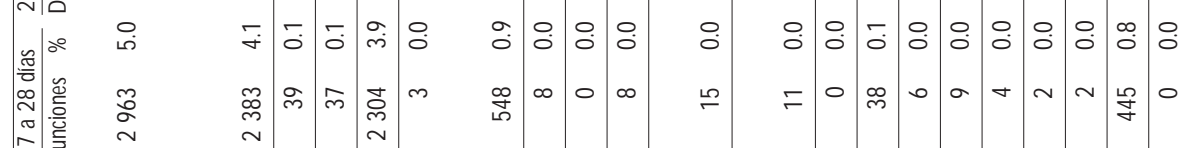

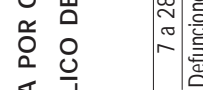

$\frac{10}{20}$

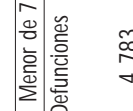

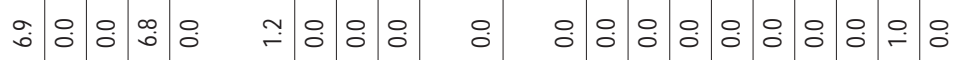

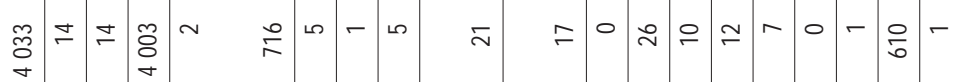

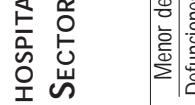

造㟧

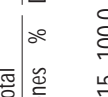

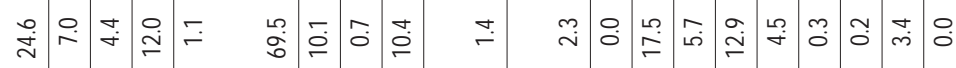

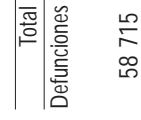




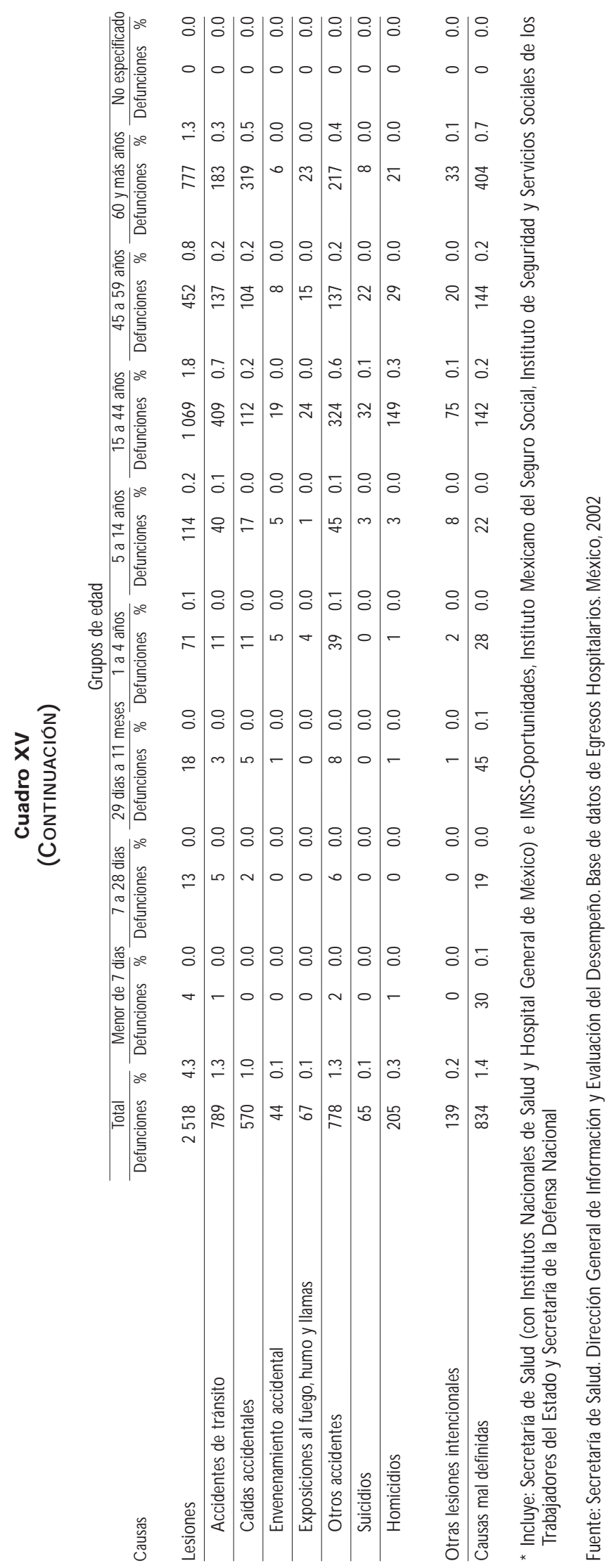




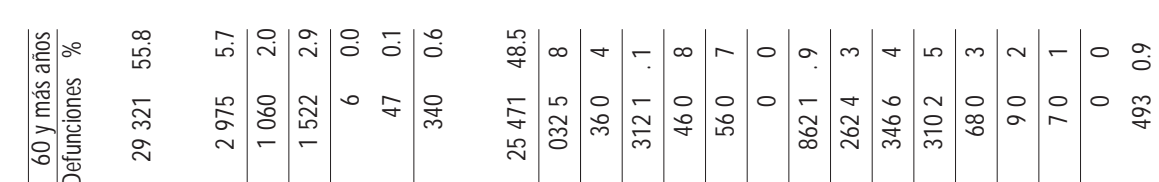

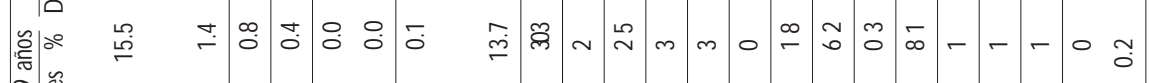

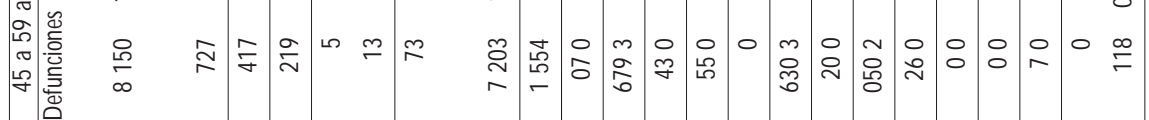

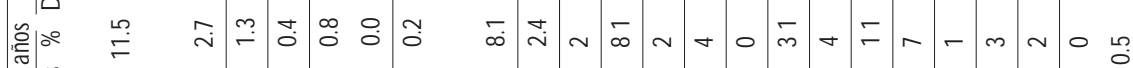

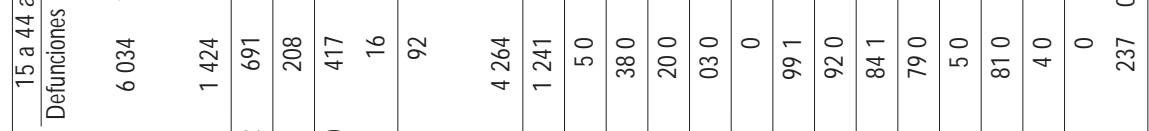

宅 $80^{\circ}$

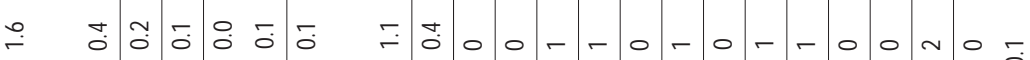

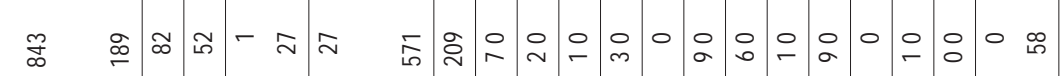

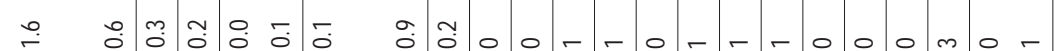

$2 *$

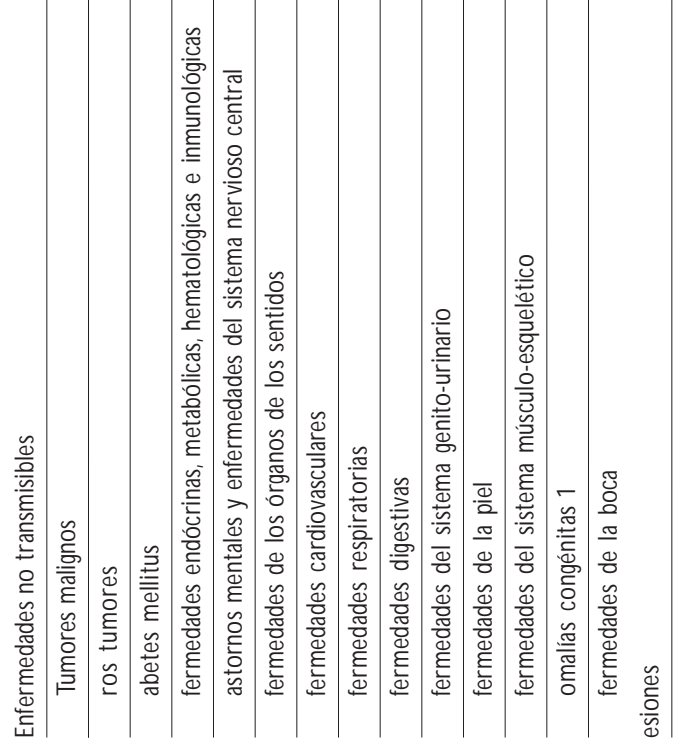




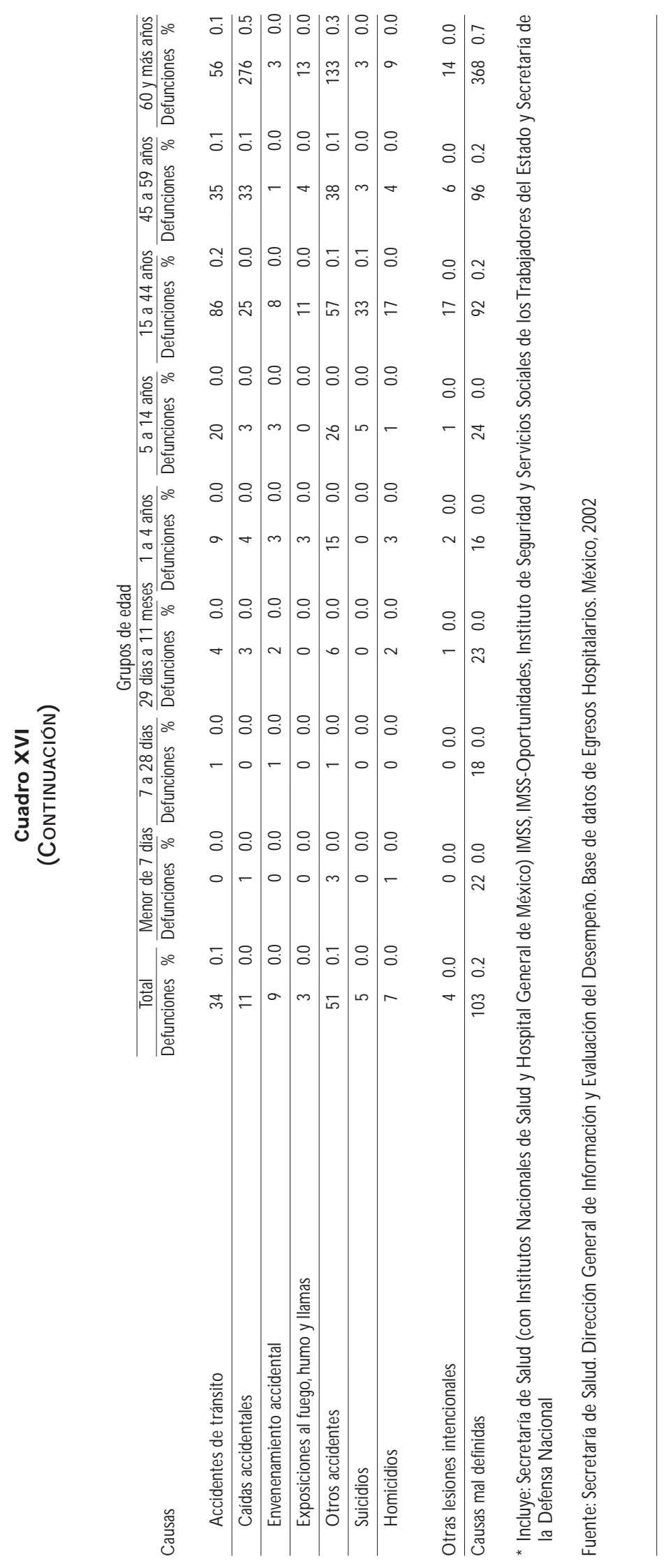

